\title{
VARIATIONS OF ACID CONCENTRATION IN DIFFER- ENT PORTIONS OF THE GASTRIC CHYME, AND ITS RELATION TO CLINICAL METHODS OF GASTRIC
} ANALYSIS *

\author{
FRANK D. GORHAM, M.D.
} ST. LOUIS

The quantitative determination of the variation of gastric acidity during different phases of digestion by the so-called "fractional method of gastric analysis" is presumably based on the assumption that the gastric chyme, after a test meal, is a homogeneous mixture, and that a small portion (from 6 to 10 c.c.), aspirated at fifteen minute intervals, represents the acid concentration of the gastric contents as a whole at that period of digestion. If this above hypothesis is correct, and if it is based on a true physiologic principle, then we should expect the acid concentration of different portions of the remaining gastric chyme to be similar at these different intervals after a test meal. It is my purpose to show that this hypothesis is not based on true physiology, and that the acidity of one portion, as obtained by the fractional method, may differ widely from the acidity of different portions of the remaining contents.

\section{METHOD OF PROCEDURE}

After the removal of the fasting contents of the stomach by means of a small, soft stomach tube of the Rehfuss type, the patient is given the Dock test meal, consisting of $30 \mathrm{gm}$. of dry shredded wheat biscuit and 400 c.c. of water. The tube is then reintroduced forty-five minutes after the meal, and the contents aspirated in 10 c.c. portions in rapid succession until the stomach is empty, the last portion being obtained after the inflation of the stomach with air, the patient lying supine. The acidity of these different portions is determined separately by the Toepfer method of analysis, titrating with tenth normal sodium hydroxid, using dimethylamidazobenzol and phenolphthalein as indicators. Tenth normal hydrochloric acid is used for hydrochloric acid deficit. An equal mixture of all samples is used for determining "the average acidity."

This method is practical and is considered sufficiently accurate for our purpose. Considerable care must be taken in preventing the patient from swallowing saliva, and also from extreme retching during the withdrawal of the gastric contents. Samples containing excess of mucus or of bile should not be used for the examination.

\footnotetext{
* From the Department of Internal Medicine, Washington University School of Medicine.
} 


\section{RESULTS OF EXPERIMENTS}

In my experiments the above method of extraction and analysis has been used in sixty-five cases with varied clinical diagnoses. In a few cases successive test meals were given, and the stomach was completely emptied at $1 / 2$ hour, 1 hour, $1 \frac{1}{2}$ and 2 hours: in the remaining cases the contents were removed at forty-five minutes after finishing the meal. In 73 per cent. of these patients the acidity of the first sample varied considerably (from 20 to 102 ) from the acidity of the

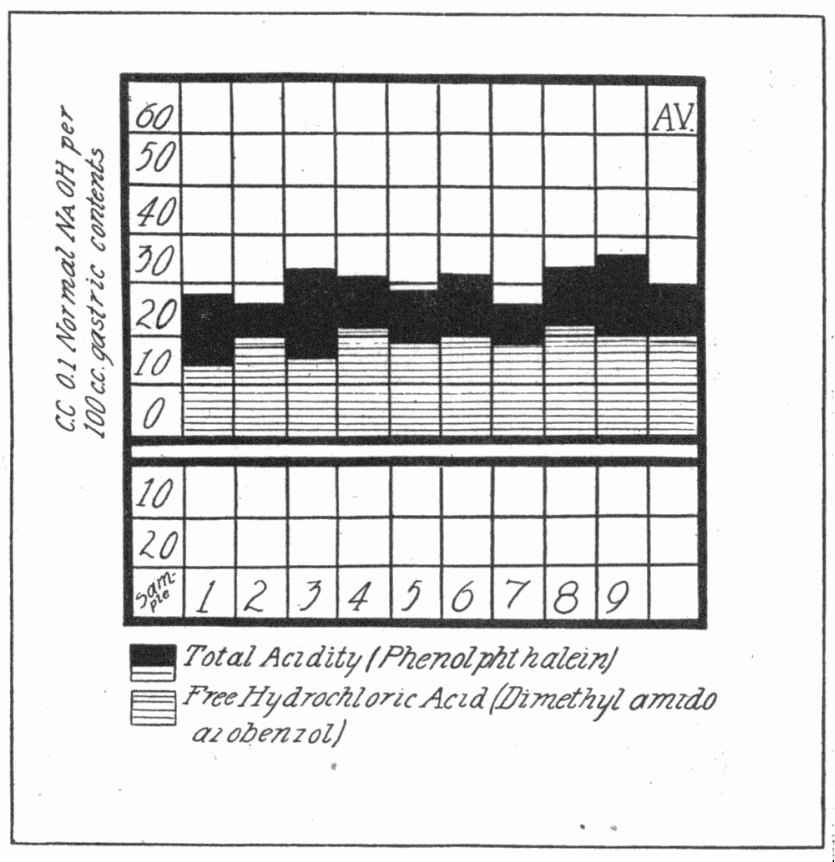

Fig. 1.-Clinical Diagnosis: Constipation. Notice the slight variation of acidity of the different portions of the gastric chyme aspirated in 10 c.c. portions in rapid succession forty-five minutes after a test meal consisting of $30 \mathrm{gm}$. shredded wheat biscuit and 400 c.c. water.

successive portions. For example, in a patient without clinical evidence of disease (Fig. 1), the acidity of the first sample obtained varied little from the acidity of the successive ones. This, however, is in marked contrast to the results obtained (Fig. 2) in a patient with a perforating duodenal ulcer (twelve hours before operation), where only a trace of free hydrochloric acid was obtained in the first sample at forty-five minutes after a test meal. One of the successive portions (Sample 11) showed a total acidity of 118 and a free hydrochloric acid 
of 106 . The average of all samples was total acidity, 66; free hydrochloric acid, 55 .

It is interesting to note that in a case of supposed achlorhydria (Fig. 3) free hydrochloric acid appeared only in one sample. The other samples, and an equal mixture of all samples, showed a free hydrochloric acid deficit.

In a case of benign pyloric stenosis, successive test meals were given, and the gastric contents were removed in 10 c.c. portions by the above method 1/2 hour, 1 hour, $11 \frac{2}{2}$ and 2 hours after the meal. The

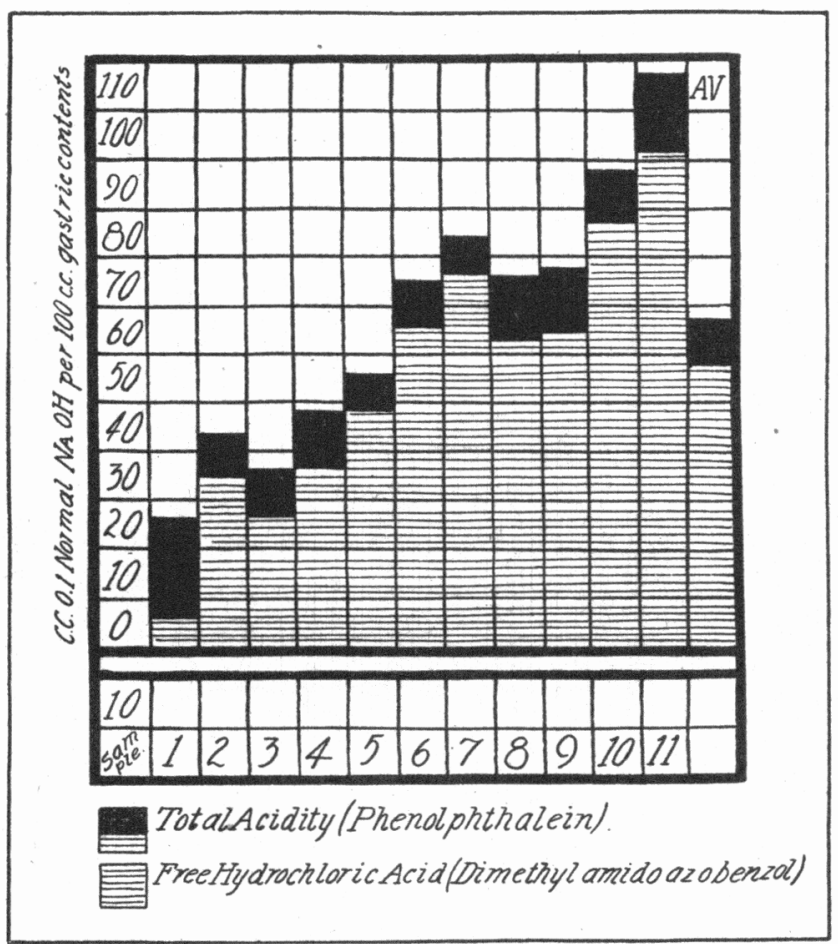

Fig. 2. - Clinical Diagnosis: Perforating duodenal ulcer (confirmed at operation). Stomach emptied by aspirating 10 c.c. portions in rapid succession forty-five minutes after a test meal consisting of $30 \mathrm{gm}$. shredded wheat biscuit and 400 c.c. water. Notice variations between "sample" (sample 1) usually obtained by the "fractional method," the high point of acid concentration (sample 11) and the average acidity of all samples (last column).

variations of the acidity of different portions of the chyme at these different periods was definite; and if we chart (Fig. 4) the low total acidity at these different periods as one curve, and the high total acidity as another curve, it will be seen how great a variation can be accounted for by the unequal acidity of different portions of the gastric chyme. 


\section{REVIEW OF LITERATURE}

Pavlov, ${ }^{1}$ in considering the movements of the stomach, was of the opinion that "the contents of the greater stomach remain for hours unmixed, and that the gastric juice digests and dissolves the mass from the exterior inwards."

According to Cannon, ${ }^{2}$ a stratification is produced which remains throughout the greater part of digestion, the mass being acted on by the gastric juice from the periphery inward. In consequence, the interior remains long unacidified and here salivary digestion may continue for hours.

Grutzner ${ }^{3}$ fed various animals with food, the successive portions of which were of different colors. At varying intervals the stomachs were tied off, removed and plunged into a salt and ice mixture; the mushy contents were frozen solid and cut into sections, always showing a definite stratification.

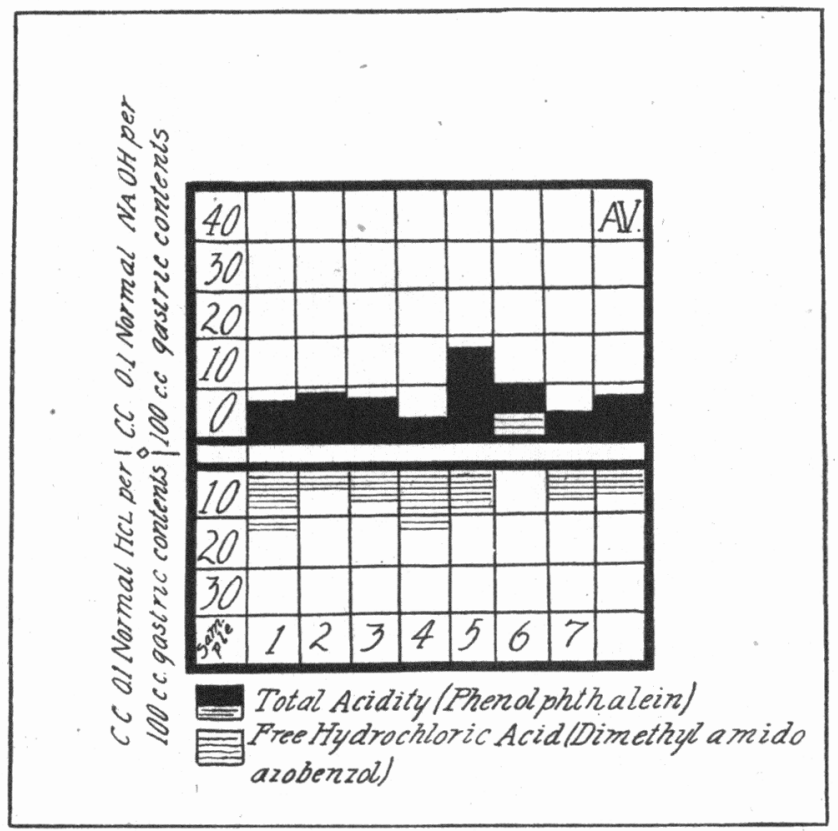

Fig. 3.-Clinical Diagnosis: Chronic Gastritis. Stomach completely emptied by aspirating 10 c.c. portions in rapid succession forty-five minutes after a test meal consisting of $30 \mathrm{gm}$. shredded wheat biscuit and 400 c.c. water-all samples except one (sample 6) and a mixture of all portions show a free hydrochloric acid deficit (last column).

Prym * investigated the behavior of a liquid meal, using Sahli's flour and butter soup. Dogs were given the soup to which blue litmus had been added, and at varying intervals the stomachs were tied off, frozen solid and cut into sections. The central portion and that near the cardia were usually found blue

1. Pavlov: The Work of the Digestive Glands, Ed. 2, London, 1910, Charles Griffin \& Co., p. 182.

2. Cannon: The Mechanical Factors of Digestion, London, 1911, Edward Arnold; New York, Longmans, Green \& Co.

3. Grutzner: Arch. f. d. ges. Physiol. $106: 463-522,1905$.

4. Prym: Deutsch. Arch. f. klin. Med. 90:310 (June) 1907. 
in color, the rest having been turned red by the hydrochloric acid. Different portions of these contents were cut out and the acidity was determined. It appeared that the degree of acidity was greater at the periphery of the mass than at the center. Prym also administered the liquid meal to a number of patients, and expressed the stomach contents through a double tube with one opening at the tip and the other $10 \mathrm{~cm}$. higher up. In this way he was able to obtain gastric contents simultaneously from different parts of the stomach and found that the two portions varied widely in acidity.

Sick," by a similar method, found that in man, after a liquid test meal (Sahli), the acidity of the pyloric gastric contents varied much from that of the fundal portion: and that, therefore, even after a liquid meal, the stomach contents are far from homogeneous.

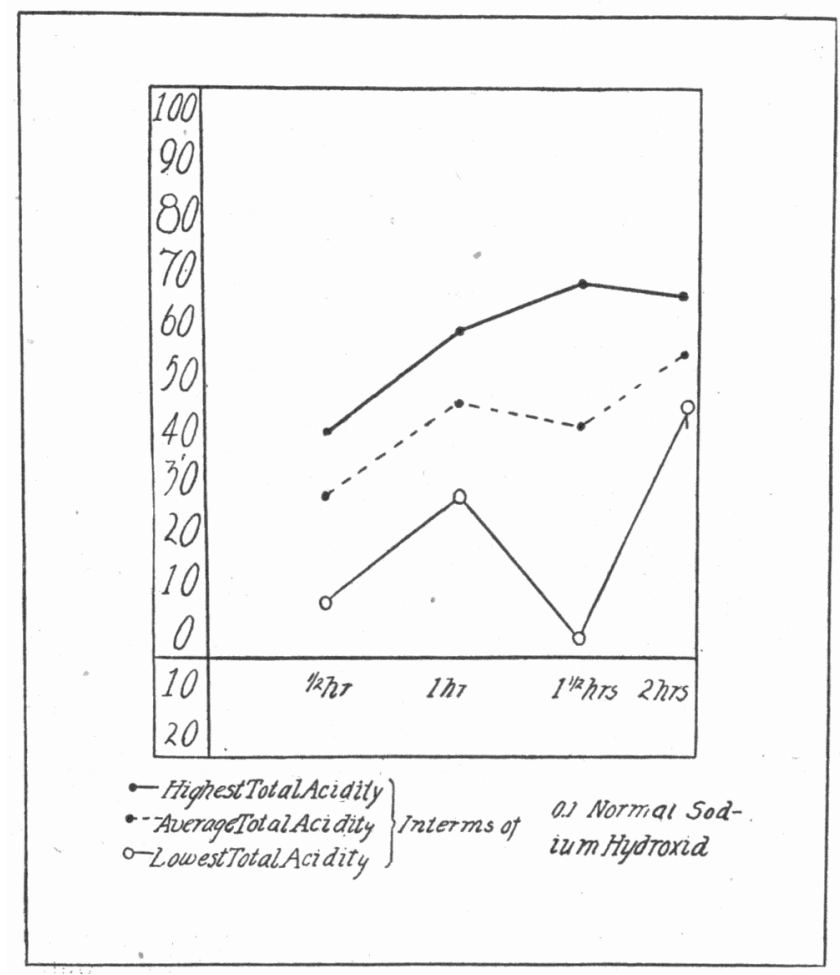

Fig. 4. - Clinical Diagnosis: Pyloric stenosis, benign. The upper curve shows the high total acidity; the lower curve shows the low total acidity, and the broken line the average total acidity of the gastric chyme aspirated in 10 c.c. portions in rapid succession at $1 / 2,1,1 / 2$ and 2 hours after successive test meals consisting of $30 \mathrm{gm}$. shredded wheat biscuit and 400 c.c. water.

Taussig ${ }^{8}$ gave a number of patients test meals and at one hour obtained all the contents possible in the erect posture, and then expressed a portion of the remainder after inflating the stomach with air, the patient lying "prone" or nearly so. The acidity of these two portions was then determined and com-

5. Sick: Ibid. 88:169 (Oct.) 1906.

6. Taussig and Rush: Boston M. \& S. J. 158:79 (Jan. 16) 1908. 
pared. He found that in a majority of instances these two portions varied in the degree of acidity. In some of the cases the difference was excessive; for instance, in a case of suspected gastric ulcer the first portion of 65 c.c. had a total acidity of 103 with free hydrochloric acid 76, while the second portion of 50 c.c. had a total acidity of 52 and free hydrochloric acid 26.

\section{DISCUSSION}

It has been shown by the result of experiments, and the work of others, that different portions of the gastric chyme may vary widely in acid concentration: and, therefore, a small sample as obtained in the "fractional method of gastric analysis," or in other methods, where only a small portion of the contents is obtained for analysis, is not, in the majority of instances, representative of the gastric chyme as a whole because the acid concentration of this portion may vary considerably from the highest or lowest acidity of some remaining portion, or the average acidity of the entire gastric contents at that period. The "sample" obtained by the "fractional method" only represents the acidity of the gastric chyme at that moment, in the part of the stomach from where it is obtained; or, in other words, it is dependent entirely on the position of the tip of the tube in the stomach. This position is necessarily a constantly changing one, due, first, to the change of size and position of the stomach while emptying itself through the pylorus and by aspiration; second, the shortening and lengthening of the stomach from gastric contraction; third, the peristaltic waves that tend to carry the tube toward the pylorus.

This explains, in part, the great variety of acidity curves obtained by the "fractional method" in the normal and similar pathologic conditions that in the past have been attributed to secretory variations. What has been said concerning the "fractional method" also applies to all other methods of gastric analyses where only a sample is obtained after a test meal. Therefore, in order to speak of quantitative gastric analysis, the stomach must be emptied completely at a definite time after a standard test meal. The true fractional analysis necessitates the giving of successive test meals and extracting them at different periods : and this, in the majority of instances, is impractical in clinical work.

\section{CONCLUSIONS}

1. A method of gastric analysis is introduced for determining the variations of acid concentration in different portions of the gastric chyme after a test meal.

2. Experimental evidence is given to show that the gastric chyme is not, in the majority of instances, a homogeneous mixture after a test meal, and that the acidity of different portions may vary widely. 
3. In the so-called "fractional" or other methods of gastric analyses where only a small sample is withdrawn, the small portion removed may or may not be representative of the gastric contents remaining in the stomach.

4. Attention is called to a physiologic principle that explains in part the great variety of curves obtained by the "fractional method" in the same individual and in similar pathologic conditions which in the past have been attributed to secretory variations.

I desire to express obligation for the technical assistance of Miss Bertha Isaacs. 